\title{
Impact of Frequency Shift on Nonlinear Compensation using Optical Phase Conjugation for M-QAM Signals
}

\author{
Nguyen Duc Binh ${ }^{1}$, Nguyen Van Dien ${ }^{2}$, Nguyen The Quang ${ }^{1}$, Nguyen Quang Nhu Quynh ${ }^{2}$, \\ Ho Phuoc Tien ${ }^{2}$, Nguyen Van Tuan ${ }^{2}$, Nguyen Tan Hung ${ }^{2}$ \\ ${ }^{1}$ Le Quy Don Technical University, Hanoi, Vietnam \\ 2 The University of Danang-University of Science and Technology, Danang, Vietnam
}

Correspondence: Nguyen Tan Hung, hung.nguyen@dut.udn.vn

Communication: received 22 May 2019, revised 24 June 2019, accepted 26 June 2019

Online publication: 23 November 2019, Digital Object Identifier: 10.21553/rev-jec.237

The associate editor coordinating the review of this article and recommending it for publication was Dr. Pham Tien Dat.

\begin{abstract}
Nonlinear compensation using optical phase conjugation (OPC) have been considered a promising technique to increase the reach of high-speed fiber-optic transmission systems. OPC-based nonlinear compensation employs an optical phase conjugation located at a middle of the fiber link to generate a complexed conjugated signal with respect to the signal in the first half of the link for propagation in the second half. OPC technique assumes a symmetry for signal propagating in the first and second half to obtain a perfect nonlinear and chromatic dispersion. However, as most of practical OPC schemes are realized by nonlinear effects such as four-wave mixing or a combination of second-harmonic generation and difference frequency generation, the frequency shift induced by OPC affects the signal symmetrical requirement for nonlinear compensation because the chromatic dispersion is different for the first and second half transmissions. In this paper, we investigate the impact of frequency shift on the nonlinear compensation using OPC for high symbol rate, high level modulation format signals. This will be important to understand the tolerance of the OPC techniques against such a practical condition for actual system implementations.
\end{abstract}

Keywords- Fiber optics communications, Optical phase conjugation, Nonlinear compensation, Four-wave mixing.

\section{INTRODUCTION}

The global data traffic has been growing exponentially over the last three decades, driven by the emerging of many bandwidth hungry applications, e.g. cloud computing, video on demand, IoT, 5G, and Big Data [1, 2]. This tremendous growth has put a lot of pressure on today's information networks where fiber-optic communication systems have been widely adopted as a backbone architecture. As a result, improving the capacity and the reach of fiber-optic transmission systems is very necessary to satisfy the ever-increasing data capacity demands. Over the last decade, a lot of research effort has been made in understanding the capacity of point-to-point optical channel [3]. However, the ultimate capacity and performance of the optical channel are still unknown due to the fiber nonlinear Kerr effects such as self-phase modulation, cross-phase modulation, and four-wave mixing [4]. These effects are particularly severer when the symbol rate, modulation format level, or/and number of channels are increased for larger data capacity transmissions.

There have been many research works focusing on removing the Kerr-induced nonlinear distortion through digital and optical compensation techniques, such as digital back propagation (DBP) $[5,6]$, optical phase conjugation (OPC) [7-11]. DBP uses digital signal processing (DSP) technology in digital coherent receiver.
However, the computing power required to conduct DBP for the transmission of multiple wavelength division multiplexing (WDM) channels is beyond the realtime computing power of the current DSP technology. In practice, the DBP is usually done for a single channel with narrow bandwidth. Therefore, the effectiveness of the DBP is still limited. Another common method is to use an OPC located in the middle of the path to compensate for nonlinear distortion [7-11]. Unlike the DBP, OPC has broadband, which can operate on multiple WDM signals simultaneously, so this technique has higher energy efficiency. Several studies have shown that, in addition to the dispersion and Kerr effect compensation, the efficiency of the OPC will increase if multiple OPCs are used on the transmission line, for example, the ability to compress the time and phase translations in the Soliton transmission system [12, 13]. Studies have also shown that distortion of the nonlinear signal phase can be reduced through the use of multiple OPCs on the pathway by the nonlinear interactions between the signal and the amplified spontaneous emission (ASE) partially compressed through the OPC layers [14].

In principle, OPC technique requires a symmetry for signal propagating in the first and second half to obtain a perfect nonlinear and chromatic dispersion $[15,16]$. However, as most of practical OPC schemes are realized by nonlinear effects such as four-wave mixing [7-11] 


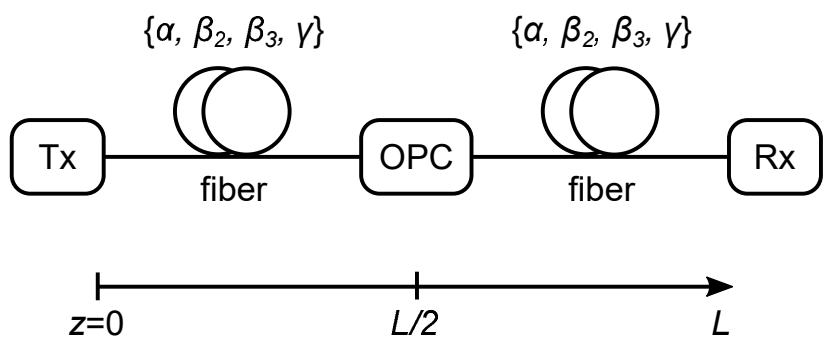

Figure 1. Schematic diagram of OPC-based transmission.

or a combination of second-harmonic generation and difference frequency generation [17], the signal is converted to another wavelength after OPC due to the nature of these nonlinear processes. Such a frequency shift induced by OPC affects the signal symmetrical requirement for nonlinear compensation because the chromatic dispersion is different for the first and second half transmissions. This effect of frequency shift due to OPC was often ignored, as most of the experiments reported so far have considered a quite low baudrate up to 50 Gbaud. However, as the symbol rate and the number of WDM channels increase, the effectiveness of OPC-based nonlinear compensation under the impact of frequency shift should be considered for the future applications of this technique for practical systems.

In this paper, we investigate the impact of frequency shift on the nonlinear compensation using OPC for high symbol rate, high level modulation format signals. At first, we present the principle of fiber nonlinear compensation using OPC. We, then, introduce the principle of OPC realization using four-wave mixing effect in fiber as well as the frequency shift caused by OPC. After that, performance of an OPC-based transmission systems is investigated through simulations under the impact of frequency shift for QPSK and 16-QAM signals. The simulation results show that while a large tolerance of OPC technique against frequency shift is obtained for signals at symbol rates below 50 Gbaud, the tolerance is quickly reduced for 100 Gbaud signal. Increasing the modulation format level from QPSK to 16-QAM also decreases the effectiveness of nonlinear compensation, however, its impact is less significant than that of increase in the symbol rate.

\section{All-Optical Nonlinear Compensation using Optical Phase Conjugation}

\subsection{Operating Principle}

Optical phase conjugation was first proposed and demonstrated for compensation of signal distortions due to chromatic dispersion and fiber nonlinearities since 1979 [18-20]. Figure 1 illustrates the schematic of a fiber-optics communication system using optical phase conjugation (OPC) for nonlinear compensation. The OPC is used to conjugate the optical signal at the mid-point of a fiber link with a purpose to reverse or compensate the signal distortions due to fiber dispersion and nonlinearity occurring in the first half of the link (span 1) through the second-half transmission (span 2). Theoretically, the nonlinear compensation process using OPC can be explained through nonlinear Schrödinger equation (NLSE) as follow:

$$
\begin{aligned}
\frac{\partial A}{\partial z}= & -\frac{\alpha(z)-g(z)}{2} A-\frac{i}{2} \beta_{2} \frac{\partial^{2} A}{\partial t^{2}} \\
& +\frac{1}{6} \beta_{3} \frac{\partial^{3} A}{\partial t^{3}}+i \gamma|A|^{2} A,
\end{aligned}
$$

where $A$ is the electric field amplitude which is a function of time, $t$, and propagation distance $z, i$ is the imaginary unit, $\alpha, g, \beta_{2}, \beta_{3}, \gamma$, respectively, are the loss coefficient, the gain coefficient, the group-velocity dispersion (GVD), the third-order dispersion (TOD), and the fiber nonlinear Kerr coefficient of the transmission link. Optical signal propagating over the first half of the link is achieved by integrating Equation (1) from the transmitter $(z=0)$ to mid-point of the link $(z=L / 2)$. In back propagation, the optical signal is given through reverse integration of Equation (1) which can be done by changing the sign of the right hand side of Equation (1) as follow [15]:

$$
\begin{aligned}
\frac{\partial A}{\partial z}= & +\frac{\alpha(z)-g(z)}{2} A+\frac{i}{2} \beta_{2} \frac{\partial^{2} A}{\partial t^{2}} \\
& -\frac{1}{6} \beta_{3} \frac{\partial^{3} A}{\partial t^{3}}-i \gamma|A|^{2} A .
\end{aligned}
$$

Equation (2), which is the time reversal of Equation (1), is not possible in practice and can only be solved numerically in the digital domain, the so-called digital back propagation. In DBP, the fiber link is divided into many small segments where the linear operation (chromatic dispersion) and nonlinear operation (fiber nonlinearity) can be treated separately [16]. Achieving the true solution of Equation (2) by DBP requires a perfect knowledge of link parameters $(\alpha, g$, $\left.\beta_{2}, \beta_{3}, \gamma\right)$ and an infinite number of segments, which consume huge amount of computation resources and large latency, especially for WDM and long-haul fiber transmissions. Alternatively, one can use optical phase conjugation in optical domain at the mid-point of a fiber link instead of DBP. Optical signal after the phase conjugation can be expressed by conjugating both sides of Equation (1) as follows. Note that since Equation (1) is generally valid, its complex-conjugated form must also be valid [15]:

$$
\begin{aligned}
\frac{\partial A^{*}}{\partial z}= & -\frac{\alpha(z)-g(z)}{2} A^{*}+\frac{i}{2} \beta_{2} \frac{\partial^{2} A^{*}}{\partial t^{2}} \\
& +\frac{1}{6} \beta_{3} \frac{\partial^{3} A^{*}}{\partial t^{3}}-i \gamma|A|^{2} A^{*},
\end{aligned}
$$

where ${ }^{*}$ denotes the complex conjugate operation. Comparing with Equation (2), we can see that Equation (3) show the back propagation of the signal through the second half of the fiber link if $\alpha$ and $\beta_{3}$ in Equation (3) have the same absolute value with different sign from those in Equation (2). In other words, under appropriate conditions discussed below, the signal distortions induced by chromatic dispersion and nonlinearity after the first half of the link can be compensated through the propagation of the phase 


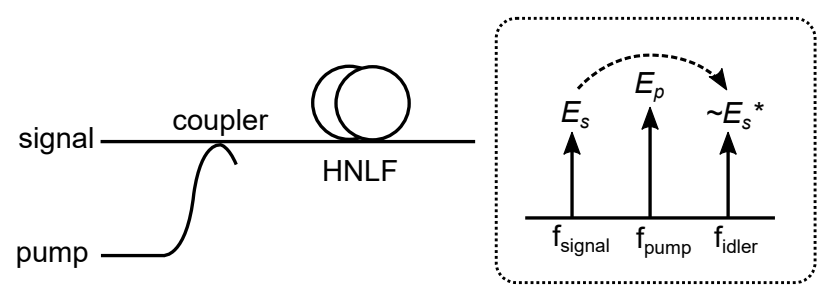

Figure 2. Configuration of OPC using FWM in HNLF.

conjugated optical signal in the second half. From Equations (2) and (3), the following link conditions should be met to achieve a perfect compensation via mid-link OPC:

$$
\begin{aligned}
& G^{(1)}(z)=G^{(2)}(L-z) \text { where } G(z)=\int_{0}^{z}[\alpha(s)-g(s)] d s, \\
& \beta_{2}^{(1)}(z)=\beta_{2}^{(2)}(L-z), \\
& \beta_{3}^{(1)}(z)=-\beta_{3}^{(2)}(L-z), \\
& \gamma^{(1)}(z)=\gamma^{(2)}(L-z),
\end{aligned}
$$

where superscripts (1) and (2) denote the first and second half of the link, respectively, and $L$ is the total distance and the OPC is located at $z=L / 2$. As a result, to perfectly cancel the loss, GVD, TOD, and Kerr nonlinearities, the dispersion and power excursion profiles along the link should be symmetrical with respect to the mid-point $(z=L / 2)$. Failure in meeting any of the above conditions would lead to the imperfect compensation of the signal distortion.

\subsection{Optical Phase Conjugation using Four-Wave Mixing}

Two optical waves are phase conjugated to each other if their complex amplitudes are conjugated with respect to the phase parameter. Generation of optical phase conjugated waves can be carried out through various optical nonlinear effects in nonlinear media such as four-wave mixing (FWM), cascaded secondharmonic generation and difference frequency generation (SHG DFG), backward stimulated scattering and so on. For applications in fiber-optics communication systems, FWM in highly nonlinear fiber (HNLF) is widely utilized to implement optical phase conjugation for its key features including format independence, waveband operation and high cascadability [21]. Figure 2 shows a configuration of OPC using FWM in HNLF. An input signal $E_{s}$ at frequency $f_{\text {signal }}$ and a pump signal $E_{p}$ at frequency $f_{\text {pump }}$ are combined by an optical coupler before launched into a HNLF. When the power of the pump signal is large enough, the degenerated FWM between $E_{s}$ and $E_{p}$ in HNLF occurs, resulting in a generation of an idler $E_{i}$ at a frequency $f_{i d l e r}$, which are formulated as follows:

$$
\begin{aligned}
E_{i} & \propto E_{p}^{2} E_{s}^{*}, \\
f_{\text {idler }} & =2 f_{\text {pump }}-f_{\text {signal }} .
\end{aligned}
$$

As a result, the idler $E_{i}$ is a phase conjugation of the input signal $E_{s}$, and can be used for the propagation

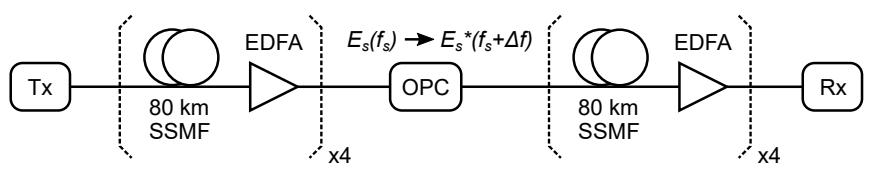

Figure 3. Configuration of OPC-based transmission system used for simulation.

through the second half of the OPC-based link. On the other hand, similarly to other techniques used for optical phase conjugation, the frequency of the conjugated idler via FWM is shifted by an amount of $\Delta f=\left|f_{\text {idler }}-f_{\text {signal }}\right|$ with respect to that of input signal as shown in Equation (6). This frequency shift violates the nonlinear compensation principle of OPC in Equation (4). Particularly, it modifies the dispersion in the second-half propagation, and thus the symmetrical properties required for OPC. The objective of this paper is to investigate the performance of the nonlinear compensation system using OPC under the impacts of frequency shift for high symbol-rate, phase modulated long-distance transmission systems.

\section{System Configuration}

Figure 3 shows configuration of the OPC-based transmission system used for numerical investigation. Simulation parameters are selected after the system in [16]. We use a widely deployed erbium-doped fiber amplifier (EDFA) amplification fiber link, consisting of 10 spans of $80 \mathrm{~km}$ single mode fiber (SMF) and an EDFA. The loss, dispersion, dispersion slope, and nonlinearity coefficients of the fiber are $\alpha=0.2 \mathrm{~dB} / \mathrm{km}, \mathrm{D}=17$ $\mathrm{ps} / \mathrm{km} / \mathrm{nm}$ (at $1550 \mathrm{~nm}$ ), $\mathrm{S}=0.075 \mathrm{ps} / \mathrm{km} / \mathrm{nm}^{2}$ (at 1550 $\mathrm{nm}$ ), and $\gamma=1.2 \mathrm{~W}^{-1} \mathrm{~km}^{-1}$, respectively. EDFA with noise figure of $6 \mathrm{~dB}$ is used to fully compensate the fiber loss of each span. An OPC is located in the middle of the link after $400 \mathrm{~km}$ transmission. Beside the function of phase conjugation, OPC also generates a frequency shift $\Delta f=\left|f_{\text {idler }}-f_{\text {signal }}\right|$ for the conjugated output signal with respect to the input signal.

In the simulation, two phase modulated signals including quadrature phase shift keying (QPSK) and 16 quadrature amplitude modulation (QAM) at symbol rates of 25 Gbaud, 50 Gbaud and 100 Gbaud at wavelength of $1550 \mathrm{~nm}$ are used. Compared to other higher order modulation formats, QPSK and 16 QAM are wellstudied in the literature for long-distance transmissions. While QPSK has been adopted to $100 \mathrm{Gbit} / \mathrm{s}$ optical transceivers for commercial optical fiber communication systems, 16-QAM is considered a candidate for the next $400 \mathrm{Gbit} / \mathrm{s}$ transceiver. At the receiver, the signal is detected by a digital coherent reception scheme with standard digital signal processing steps such as carrier phase and frequency recovery, timing recovery, and chromatic dispersion compensation (DC). Error vector magnitude (EVM) value, bit error rate (BER), and $Q$ factor of the detected signal are calculated from the 
received constellation diagram as follows [22]:

$$
\begin{gathered}
E V M=\frac{\frac{1}{N} \sum_{n=1}^{N}\left\|S_{n}-S_{0, n}\right\|^{2}}{\frac{1}{N} \sum_{n=1}^{N}\left\|S_{0, n}\right\|^{2}}, \\
B E R=\frac{\left(1-M^{-1 / 2}\right)}{\frac{1}{2} \log _{2} M} \cdot \operatorname{erfc}\left[\sqrt{\frac{\frac{3}{2}}{(M-1) E V M^{2} \cdot k^{2}}}\right], \\
Q=20 \lg (\sqrt{2}(\operatorname{erfcinv}(2 B E R))),
\end{gathered}
$$

where $S_{n}$ is the normalized $n$th symbol in the stream of measured symbols, $S_{0, n}$ is the ideal normalized constellation point of the $n$th symbol, $N$ is the number of unique symbols in the constellation, $M$ is the number of points on the signal constellation. $k$ is the coefficient depending on the type of modulation, and calculated according to the table below:

Table I

The Calculation of $k$ with Some Modulation

\begin{tabular}{ccccc}
\hline Format: & QPSK & 16QAM & 32QAM & 64QAM \\
k: & 1 & $\sqrt{9 / 5}$ & $\sqrt{17 / 10}$ & $\sqrt{7 / 3}$ \\
\hline
\end{tabular}

\section{Results And Discussion}

The performance of nonlinear compensation using OPC is investigated through two system scenarios including single-channel and multiple-channel transmissions of high-baudrate, quadrature phase shift keying (QPSK) signals. While the performance of signal-channel systems provides a baseline to understand and compare the effect of individual factors to the system, the multiple-channel transmission scenario brings compound impact on the effectiveness of OPC for higher capacity wavelength-division multiplexed systems.

\subsection{Single-channel OPC-based Transmissions}

To meet the ever-increasing capacity demands in an economically attractive manner, the data rate per WDM channel has been increasing exponentially over the last two decades [23]. Single channel data rate can be increased by pushing both the symbol rate (50 Gbaud or higher) and the modulation format order of M-quadrature amplitude modulation (M-QAM), e.g. QPSK and 16-QAM [24], which in principle, require significant improvement in hardware implementation, e.g. electronic bandwidth, for higher signal-to-noise ratio. Even though the OPC-based nonlinear compensation is considered a promising technique to improve the transmission reach for such high-baudrate, advanced modulation format signals, its effectiveness under the practical condition of OPC, here, the frequency shifting effect, is one of the important issues to investigate.

4.1.1 Quadrature Phase Shift Keying (QPSK): Figures 4 and 5 show the performance of QPSK signal at 50 Gbaud and 100 Gbaud, respectively, after $800 \mathrm{~km}$ SSMF transmission as functions of the launched power. Frequency shifts of $300 \mathrm{GHz}$ and $600 \mathrm{GHz}$ are applied

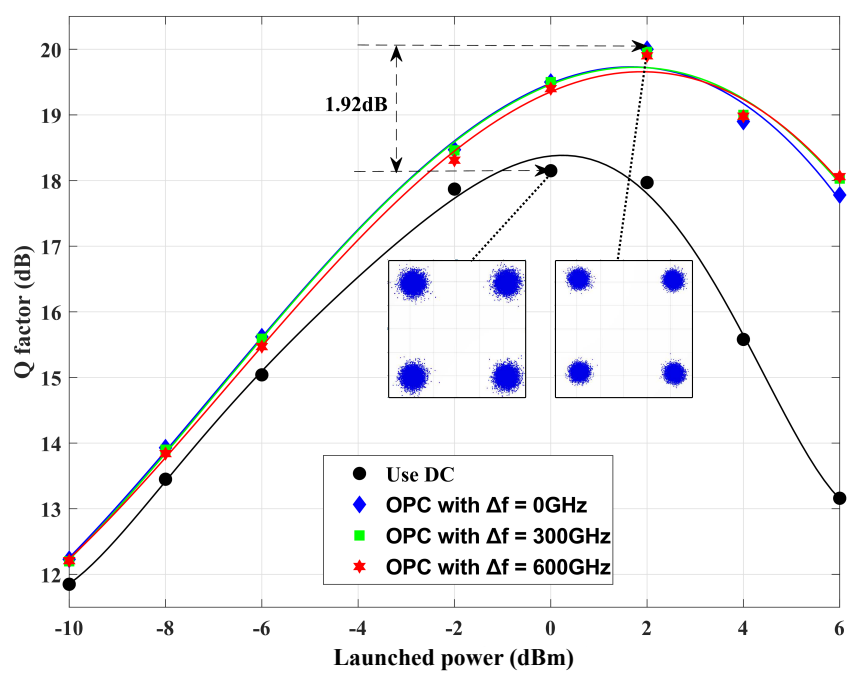

Figure 4. Q factor of 50 Gbaud QPSK signal as a function of launched power in single-channel OPC-based $800 \mathrm{~km}$ transmission. Insets are constellations at nonlinear thresholds.

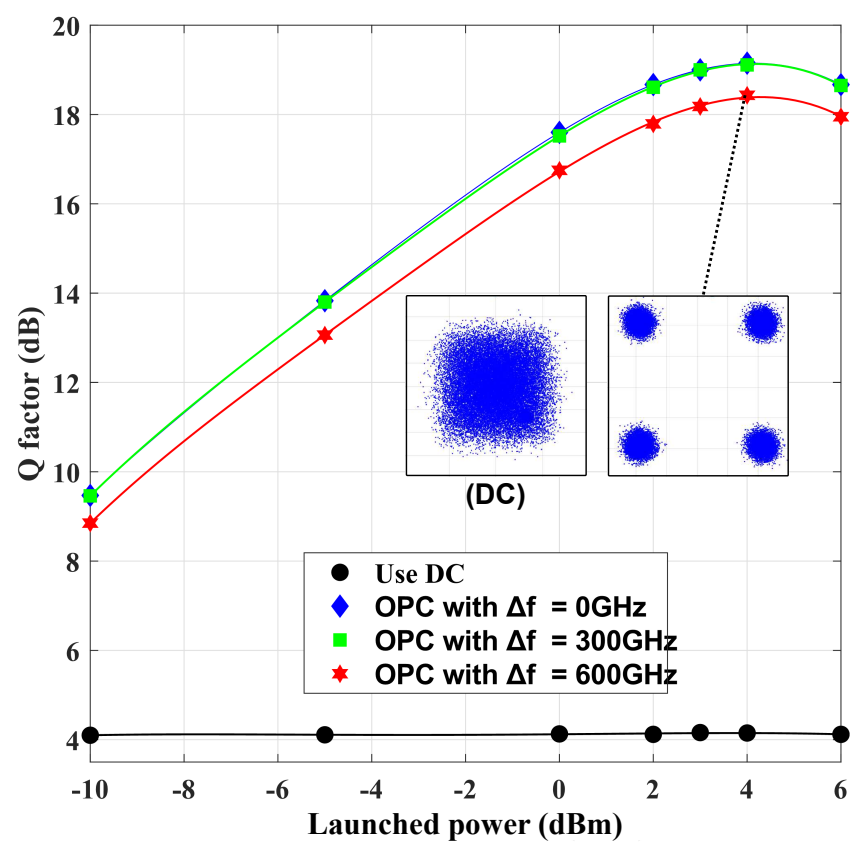

Figure 5. Q factor of 100 Gbaud QPSK signal as a function of launched power in single-channel OPC-based $800 \mathrm{~km}$ transmission. Insets are constellations at nonlinear thresholds.

for the signal after OPC. Use DC stands for the use of dispersion compensation only without OPC. We can see that there is an optimal launched power for each case of transmission. This level is called the nonlinear threshold, where the fiber nonlinear effects overwhelmed the optical SNR increase due to the increasing of launched power. For 50 Gbaud QPSK signal $(100 \mathrm{~Gb} / \mathrm{s}$ data rate), the nonlinear threshold is increased from $0 \mathrm{dBm}$ to $2 \mathrm{dBm}$, making the $\mathrm{Q}$ factor increased by $1.92 \mathrm{~dB}$ through the use of OPC with respect to the case of dispersion compensation only. Insets of Figure 4 are constellations of the 50 Gbaud QPSK signal in the case without and with the use of OPC. For 100 Gbaud QPSK signal (200 Gb/s data rate), it is almost not possible to transmit the signal over $800 \mathrm{~km}$ SSMF by using only 


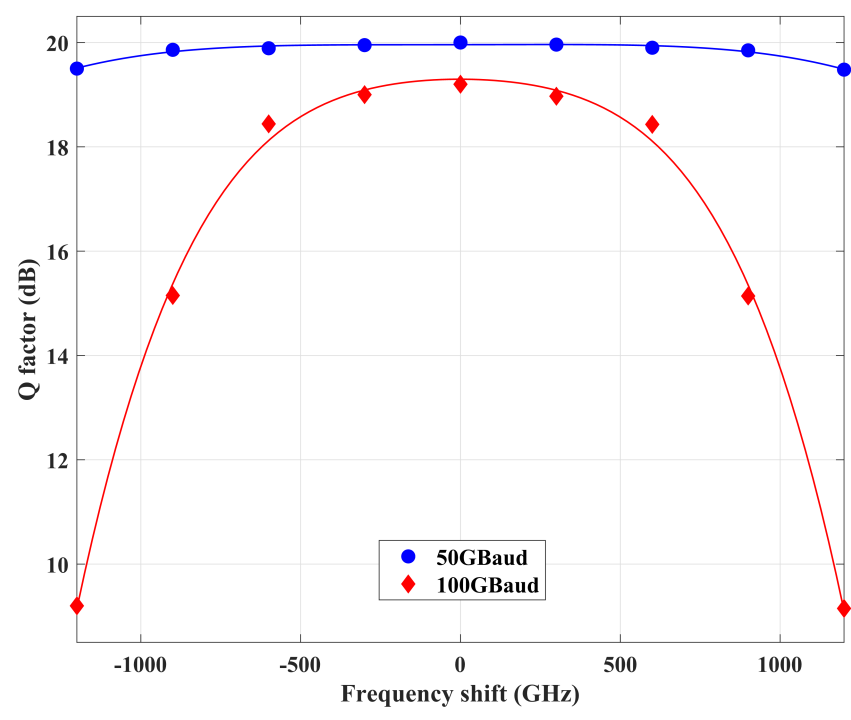

Figure 6. Tolerance of OPC-based nonlinear compensation against frequency shift for single-channel QPSK $800 \mathrm{~km}$ transmission.

dispersion compensation because the signal at such a high baudrate requires high optical signal to noise ratio (OSNR), but increasing the launched power was not possible due to fiber nonlinearities. On the other hand, the use of OPC significantly improves the $Q$ factor to over $18 \mathrm{~dB}$ (see constellations in the insets of Figure 5). These results clearly show the effect of nonlinear compensation by using OPC, especially for high symbol rate signal. As for the effect of frequency shifts after OPC, the performance differences between $0 \mathrm{GHz}, 300 \mathrm{GHz}$ and $600 \mathrm{GHz}$ shifting are negligible for 50 Gbaud QPSK signal while there is a small degradation for 100 Gbaud QPSK signal at $600 \mathrm{GHz}$ frequency shift.

To further investigate the tolerance of the nonlinear compensation using OPC technique for QPSK signal, we evaluate the transmission performance over $800 \mathrm{~km}$ SSMF as a function of OPC-induced frequency shift as shown in Figure 6. We can see that the performance of 50 Gbaud QPSK signal has a strong tolerance against the frequency shift, in which the $\mathrm{Q}$ factor degrades by less than $1 \mathrm{~dB}$ within $\pm 1200 \mathrm{GHz}$ frequency shift range. On the other hand, the 100 Gbaud QPSK signal shows a strong tolerance within $\pm 600 \mathrm{GHz}$ frequency shift range. Beyond that range, the transmission performance of OPC system is quickly degraded. These results can be explained that at high symbol rates, e.g. 100 Gbaud, the signal is very sensitive to chromatic dispersion, the signal symmetry with respect to the middle point of the link is no longer maintained due to the frequency shift by OPC. Therefore, the effectiveness of nonlinear compensation using OPC is quickly degraded for high symbol rate signal. The lower symbol rate signals, e.g. $50 \mathrm{Gbaud}$, on the other hand, is quite tolerant to chromatic dispersion, and thus it is also tolerant to the frequency shift by OPC.

4.1.2 16-Quadrature Amplitude Modulation (16-QAM): M-Quadrature amplitude modulation (M-QAM) is one of the advanced modulation formats to improve the spectral efficiency, increasing the data rate while keep-

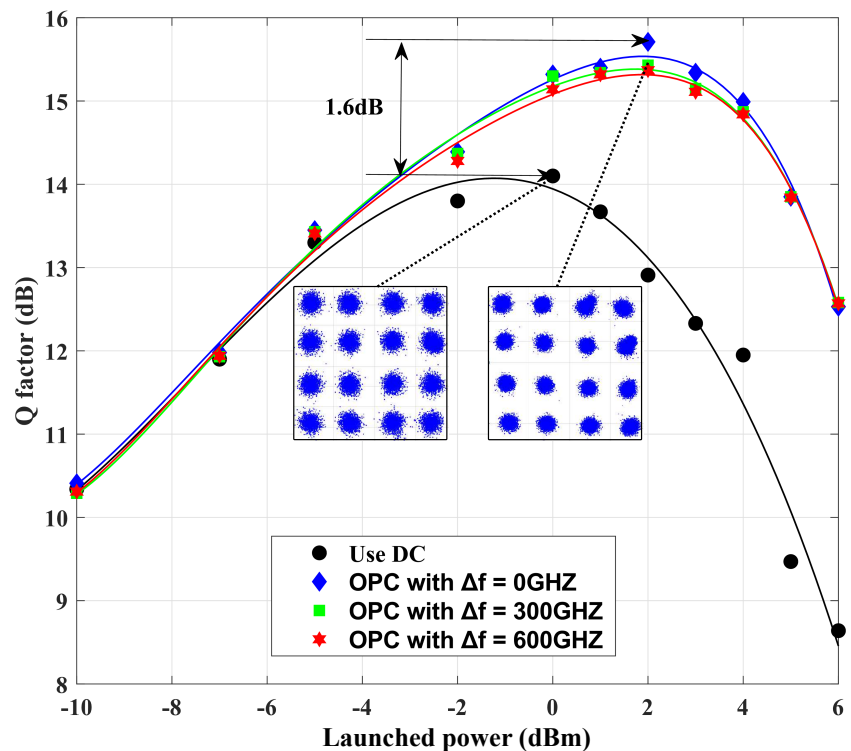

Figure 7. Q factor of 25 Gbaud 16-QAM signal as a function of launched power in single-channel OPC-based $800 \mathrm{~km}$ transmission. Insets are constellations at nonlinear thresholds.

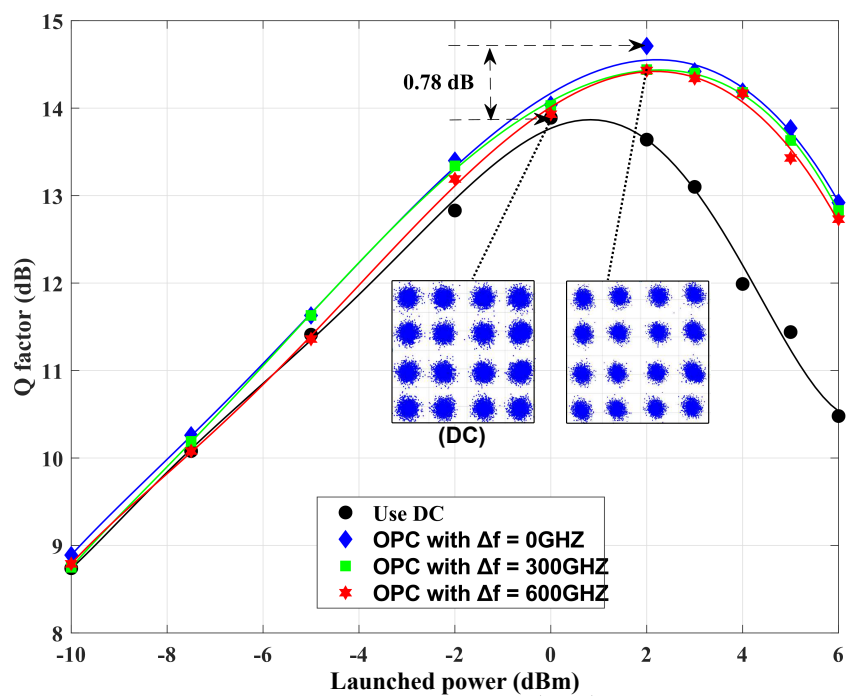

Figure 8. Q factor of 50 Gbaud 16-QAM signal as a function of launched power in single-channel OPC-based $800 \mathrm{~km}$ transmission. Insets are constellations at nonlinear thresholds.

ing the same spectral occupation. For example, using 16-QAM increases the data rate by 4 times. Figures 7 and 8 present $800 \mathrm{~km}$ transmission performance of 16QAM signal as functions of the launched power for 25 Gbaud (100 Gb/s) and 50 Gbaud (200 Gb/s), respectively. Similarly, we also apply different frequency shifts of $300 \mathrm{GHz}$ and $600 \mathrm{GHz}$ for the signal after OPC. As can be seen from Figures 7 and 8, using OPC helps to improve the nonlinear threshold from $0 \mathrm{dBm}$ to 2 $\mathrm{dBm}$, and also improve the $\mathrm{Q}$ factor by $1.6 \mathrm{~dB}$ and 0.78 $\mathrm{dB}$ with respect to the case without using OPC (only dispersion compensation) for 25 Gbaud and 50 Gbaud signals, respectively. Such improvements can also be seen through the improvement in the constellations as shown in the insets of Figures 7 and 8. In addition, we can see that the adding of frequency shifts of $300 \mathrm{GHz}$ and $600 \mathrm{GHz}$ to the signal after OPC has a small impact 


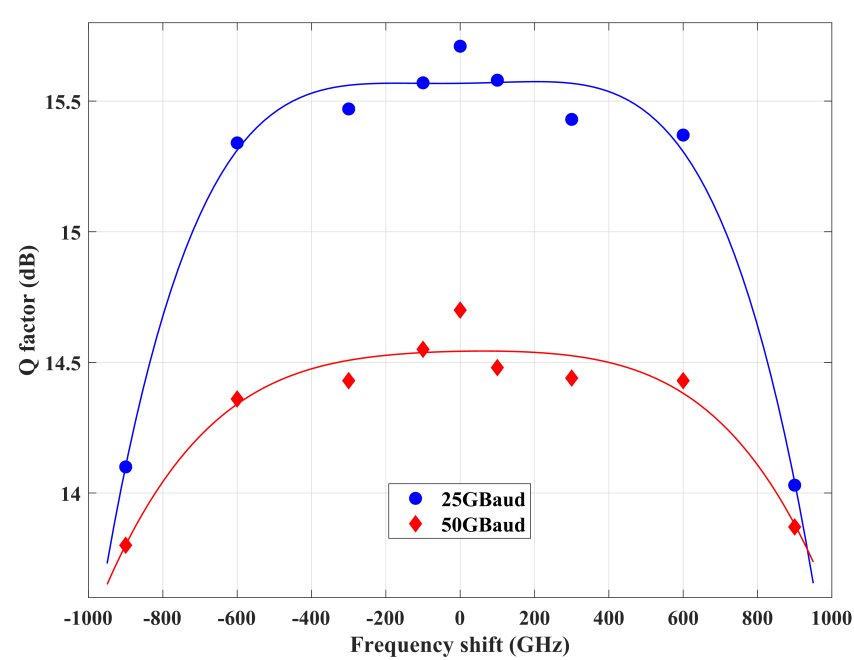

Figure 9. Tolerance of OPC-based nonlinear compensation against frequency shift for single-channel 16-QAM $800 \mathrm{~km}$ transmission.

on the transmission performance and the nonlinear compensation.

Next, the tolerance of the nonlinear compensation using OPC against the frequency shift is further investigated for 25 Gbaud and 50 Gbaud signals. The results are shown in Figure 9. We can see that the 25 Gbaud and 50 Gbaud signals both exhibit a strong tolerance to the frequency shift by OPC. Within $\pm 600 \mathrm{GHz}$ frequency shift range, $\mathrm{Q}$ factor varies within a small range of $0.5 \mathrm{~dB}$ for both cases. Such frequency shift tolerances come from the fact that at low symbol rates, e.g. 25 Gbaud or 50 Gbaud, the signals are less sensitive to the chromatic dispersion. The dispersion of these signals before and after OPC is not significantly changed for the frequency shift within $\pm 600 \mathrm{GHz}$. As a result, the effectiveness of nonlinear compensation using OPC is maintained. In addition, compared with the signal of the same symbol rate at 50 Gbaud (see Figures 6 and 9), the increase in the modulation format level from 4 (QPSK) to 16 (M-QAM) reduces the tolerance of OPCbased transmission against frequency shift. However, the impact of increase in the modulation format level is less significant than that of increase in the symbol rate (see Figure 6). Therefore, for OPC-based transmission system, it is recommended to increase the data rate of a single channel by pushing the modulation format level (M-QAM) rather than by increasing the symbol rate due to the strong effect of chromatic dispersion on high symbol rate signals.

\subsection{Multiple-Channel Transmissions}

In this section, we investigate the impact of frequency shift on multiple-channel OPC-based nonlinear compensation. Four channels at frequencies of $193.1 \mathrm{THz}$ (channel 1), $193.2 \mathrm{THz}$ (channel 2), $193.3 \mathrm{THz}$ (channel 3), and $193.4 \mathrm{THz}$ (channel 4) are wavelength-division multiplexed and transmitted through the OPC-based transmission system in Figure 3. The transmission distance is $800 \mathrm{~km}$. Figures 10 and 11 shows Q factor of four channels at the receiver sides for 50 Gbaud QPSK

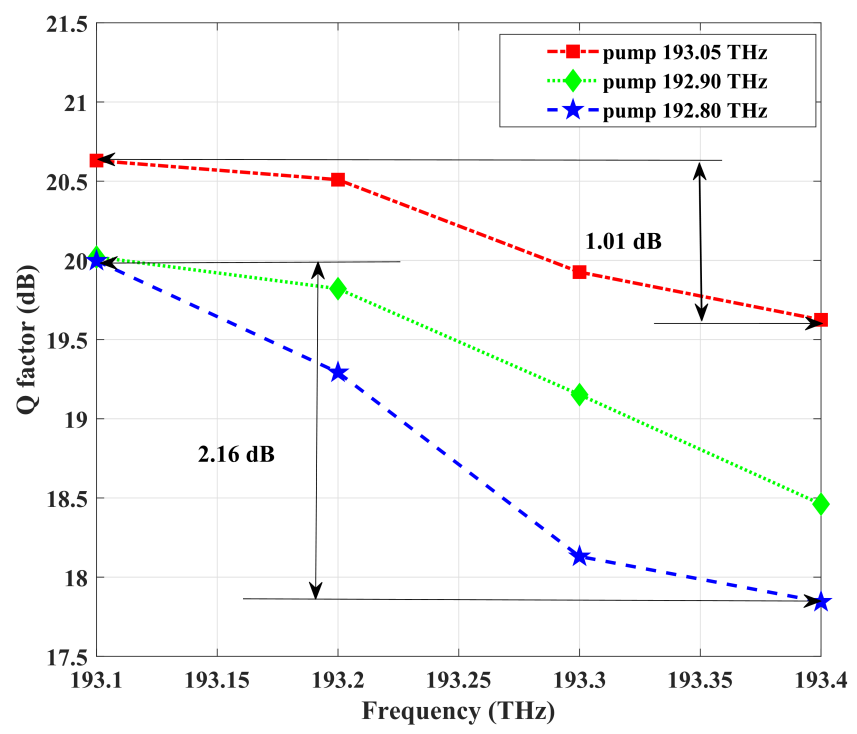

Figure 10. Q factor of four WDM 50 Gbaud QPSK channels after 800 $\mathrm{km}$ OPC-based transmission at different pump frequency settings.

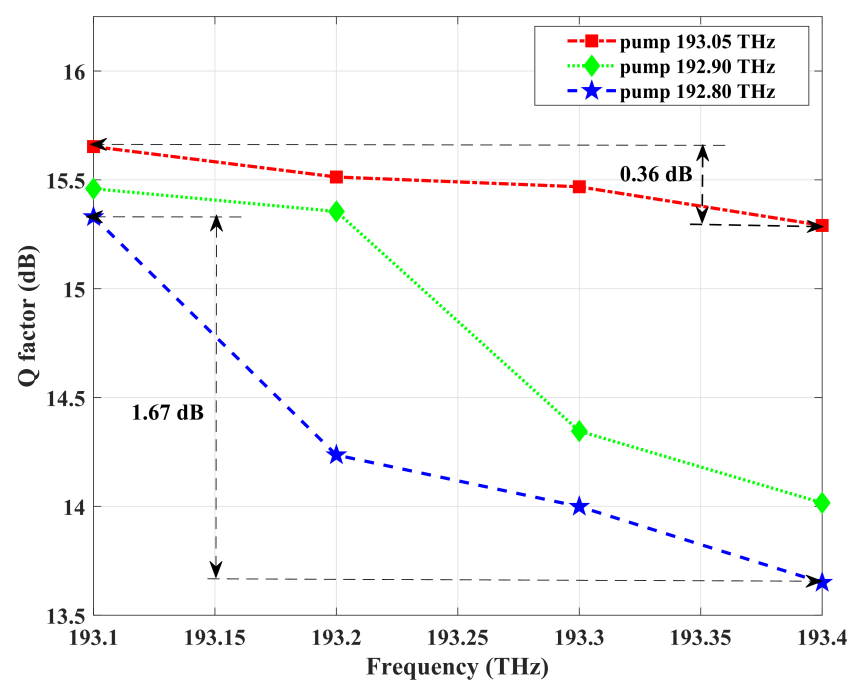

Figure 11. Q factor of four WDM 25 Gbaud 16-QAM channels after $800 \mathrm{~km}$ OPC-based transmission at different pump frequency settings.

(100 Gb/s) and 25 Gbaud 16-QAM (100 Gb/s) signals, respectively. Four cases of OPC pump frequency settings: $193.05 \mathrm{THz}, 192.9 \mathrm{THz}$ and $192.8 \mathrm{THz}$ are considered. Frequencies of the converted channels after OPC are different, following Equation (6). Therefore, different frequency shifts are induced for each WDM channels, in which the further to the pump the channel is located, the larger the frequency shift $\Delta f$ becomes after OPC. As can be seen in Figures 10 and 11, Q factor is gradually degraded for channels located further to the pump because the larger frequency shift degrades the effectiveness of nonlinear compensation using OPC.

Furthermore, when the pump is set further to the WDM channels, Q factors of the received WDM channels are decreased, and the Q-factor variation between the best and worst channels is also increased. For 50 Gbaud QPSK signal the Q-factor variation increases from $1.01 \mathrm{~dB}$ to $2.16 \mathrm{~dB}$ when the pump frequency increases from $193.05 \mathrm{THz}$ to $192.8 \mathrm{THz}$ (see Figure 10). 
For 25 Gbaud 16-QAM signal, as seen in Figure 11 the Q-factor variation is $0.36 \mathrm{~dB}$ and $1.67 \mathrm{~dB}$ for pump frequency of $193.05 \mathrm{THz}$ and $192.8 \mathrm{THz}$, respectively. These results are due to the fact that at lower symbol rate the impact of frequency shift on the OPCbased nonlinear compensation is smaller because the lower symbol rate signal is less sensitive to chromatic dispersion. These results are consistent to the ones in Section 4.1 when considering the effectiveness of nonlinear compensation on factors such as symbol rate and modulation format order.

\section{Conclusion}

We have presented the impact of frequency shift on the nonlinear compensation using OPC for high symbol rate, high level modulation format signals. Simulations have been carried out to investigate the performance of an OPC-based transmission systems using QPSK and 16-QAM signals. The simulation results confirm the benefits of OPC in compensating the signal distortions due to fiber nonlinear and chromatic dispersion. It also shows a large tolerance of OPC technique against frequency shift for signals at symbol rates below 50 Gbaud. However, the tolerance is degraded for higher symbol rate signal at $100 \mathrm{Gbaud}$. Increasing the modulation format level from QPSK to 16-QAM also causes degradation of the effectiveness of nonlinear compensation, however, its impact is less significant than that of increase in the symbol rate.

\section{ACKNOWLEDGMENTS}

This research is funded by Vietnam National Foundation for Science and Technology Development (NAFOSTED) under grant number 102.04-2016.36.

\section{REFERENCES}

[1] J. Gantz and D. Reinsel, "The digital universe in 2020: Big data, bigger digital shadows, and biggest growth in the far east," IDC IVIEW: IDC Analyze the future, no. 2012, pp. 1-16, 2012.

[2] Cisco Systems, "Cisco visual networking index: Forecast and trends, 2017-2022," White Paper, 2018.

[3] A. D. Ellis, M. E. McCarthy, M. A. Z. Al Khateeb, M. Sorokina, and N. J. Doran, "Performance limits in optical communications due to fiber nonlinearity," Advances in Optics and Photonics, vol. 9, no. 3, pp. 429-503, 2017.

[4] G. P. Agrawal, Nonlinear Fiber Optics, 2nd ed. Academic Press, San Diego, USA, 1995.

[5] E. Ip and J. M. Kahn, "Compensation of dispersion and nonlinear impairments using digital backpropagation," Journal of Lightwave Technology, vol. 26, no. 20, pp. 3416$3425,2008$.

[6] E. Temprana, E. Myslivets, L. Liu, V. Ataie, A. Wiberg, B. Kuo, N. Alic, and S. Radic, "Two-fold transmission reach enhancement enabled by transmitter-side digital backpropagation and optical frequency comb-derived information carriers," Optics Express, vol. 23, no. 16, pp. 20 774-20 783, 2015.

[7] I. Phillips, M. Tan, M. Stephens, M. E. McCarthy, E. Giacoumidis, S. Sygletos, P. Rosa, S. Fabbri, S. T. Le, T. Kane- san, S. K. Turitsyn, N. J. Doran, P. Harper, and A. D. Ellis, "Exceeding the nonlinear-Shannon limit using Raman laser based amplification and optical phase conjugation," in Proceedings of the OFC 2014. IEEE, 2014, pp. 1-3.

[8] K. Solis-Trapala, M. Pelusi, H. N. Tan, T. Inoue, and S. Namiki, "Optimized WDM transmission impairment mitigation by multiple phase conjugations," Journal of Lightwave Technology, vol. 34, no. 2, pp. 431-440, 2015.

[9] S. Yoshima, Z. Liu, Y. Sun, K. R. Bottrill, F. Parmigiani, P. Petropoulos, and D. J. Richardson, "Nonlinearity mitigation for multi-channel 64-QAM signals in a deployed fiber link through optical phase conjugation," in Proceedings of the Optical Fiber Communications Conference and Exhibition (OFC). IEEE, 2016, pp. 1-3.

[10] A. D. Ellis, M. Tan, M. A. Iqbal, M. A. Z. Al-Khateeb, V. Gordienko, G. S. Mondaca, S. Fabbri, M. F. Stephens, M. E. McCarthy, A. Perentos et al., " $4 \mathrm{~Tb} / \mathrm{s}$ transmission reach enhancement using $10 \times 400 \mathrm{~Gb} / \mathrm{s}$ super-channels and polarization insensitive dual band optical phase conjugation," Journal of Lightwave Technology, vol. 34, no. 8, pp. 1717-1723, 2016.

[11] H. Hu, R. M. Jopson, A. H. Gnauck, S. Randel, and S. Chandrasekhar, "Fiber nonlinearity mitigation of WDM-PDM QPSK/16-QAM signals using fiber-optic parametric amplifiers based multiple optical phase conjugations," Optics Express, vol. 25, no. 3, pp. 1618-1628, 2017.

[12] W. Forysiak and N. Doran, "Reduction of Gordon-Haus jitter in soliton transmission systems by optical phase conjugation," Journal of Lightwave Technology, vol. 13, no. 5, pp. 850-855, 1995.

[13] C. McKinstrie, S. Radic, and C. Xie, "Reduction of soliton phase jitter by in-line phase conjugation," Optics Letters, vol. 28, no. 17, pp. 1519-1521, 2003.

[14] M. H. Shoreh, "Compensation of nonlinearity impairments in coherent optical OFDM systems using multiple optical phase conjugate modules," Journal of Optical Communications and Networking, vol. 6, no. 6, pp. 549-558, 2014.

[15] S. Namiki, K. Solis-Trapala, N. T. Hung, M. Pelusi, and T. Inoue, "Multi-channel cascadable parametric signal processing for wavelength conversion and nonlinearity compensation," Journal of Lightwave Technology, vol. 35, no. 4, pp. 815-823, 2016.

[16] T. L. Son, N. T. Hung, and H. Buelow, "Imperfection induced bandwidth limitation in nonlinearity compensation," in Proceedings of the European Conference on Optical Communication (ECOC). IEEE, 2017, pp. 1-3.

[17] T. Kobayashi, T. Umeki, R. Kasahara, H. Yamazaki, M. Nagatani, H. Wakita, H. Takenouchi, and Y. Miyamoto, "96-Gbaud PDM-8QAM Single Channel Transmission over $9,600 \mathrm{~km}$ by Nonlinear Tolerance Enhancement using PPLN-based Optical Phase Conjugation," in Proceedings of the Optical Fiber Communications Conference and Exposition (OFC). IEEE, 2018, pp. 1-3.

[18] A. Yariv, D. Fekete, and D. M. Pepper, "Compensation for channel dispersion by nonlinear optical phase conjugation," Optics Letters, vol. 4, no. 2, pp. 52-54, 1979.

[19] S. Watanabe, T. Chikama, G. Ishikawa, T. Terahara, and H. Kuwahara, "Compensation of pulse shape distortion due to chromatic dispersion and Kerr effect by optical phase conjugation," IEEE Photonics Technology Letters, vol. 5, no. 10, pp. 1241-1243, 1993.

[20] C. Lorattanasane and K. Kikuchi, "Design theory of longdistance optical transmission systems using midway optical phase conjugation," Journal of Lightwave Technology vol. 15, no. 6, pp. 948-955, 1997.

[21] N. T. Hung, T. Inoue, K. Solis-Trapala, S. Petit, Y. Oikawa, K. Ota, S. Takasaka, T. Yagi, M. Pelusi, and S. Namiki, "On the cascadability of all-optical wavelength converter for high-order QAM formats," Journal of Lightwave Technology, vol. 34, no. 13, pp. 3194-3205, 2016. 
[22] W. Freude, R. Schmogrow, B. Nebendahl, M. Winter, A. Josten, D. Hillerkuss, S. Koenig, J. Meyer, M. Dreschmann, M. Huebner et al., "Quality metrics for optical signals: eye diagram, Q-factor, OSNR, EVM and BER," in Proceedings of the 14th International Conference on Transparent Optical Networks (ICTON). IEEE, 2012, pp. $1-4$.

[23] P. J. Winzer and D. T. Neilson, "From scaling disparities to integrated parallelism: A decathlon for a decade," Journal of Lightwave Technology, vol. 35, no. 5, pp. 10991115, 2017.

[24] P. J. Winzer, "High-spectral-efficiency optical modulation formats," Journal of Lightwave Technology, vol. 30, no. 24, pp. 3824-3835, 2012.

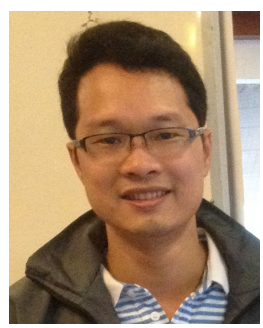

Nguyen Duc Binh was born in 1984. He received B.E. degree from Le Quy Don Technical University, Viet Nam, in 2008, and M.E. degree in 2013 from Le Quy Don Technical University, Viet Nam. From 2013 to 2015, He has worked as an operator and manager engineer of optical transmission network at the Hightech Communication Center, Communications Command. He is currently a Ph.D. student at Le Quy Don Technical University, Hanoi, Vietnam.

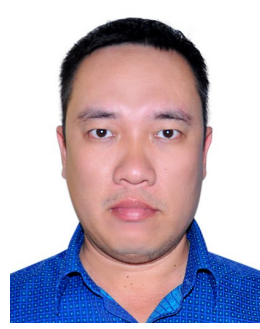

Nguyen Van Dien received the B.E. degree from Ho Chi Minh City University of Technology and the Diplom-Ingenieur degree from University of Stuttgart, Germanyin 2003 and 2011, respectively. He is now a Ph.D. candidate at The Univeristy of Danang - University of Science and Technology. His research interests includefiber-optics communications, radio-over-fiber and microwave photonics for next-generation access networks.

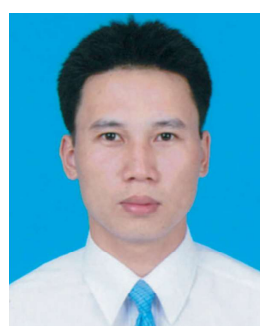

Nguyen The Quang was born in 1978. He received the B.E. degree from National Defense Academy, Japan, in 2004, the M.E. and Ph.D. degrees from the University of Electro-Communications, Tokyo, Japanin 2009 and 2012, respectively. From 2012 to 2014, he worked as a postdoctoral fellow at the Department of Communication Engineering and Informatics, the University of ElectroCommunications, Tokyo, Japan. He was a recipient of the Young Scientist Award at the 15th OptoElectronics and Communications Conference (OECC 2010) presented by the IEEE Photonics Society Japan Chapter. His research interest is all-optical signal processing based on nonlinear fiber optics for WDM and OTDM systems. He is currently a Lecturer at Le Quy Don Technical University, Hanoi, Vietnam.

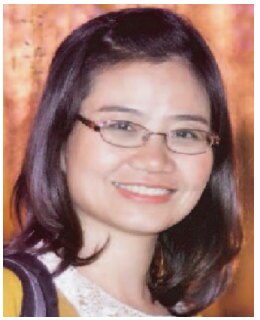

Nguyen Quang Nhu Quynh received the B.E. and M.E. degrees from The University of Danang - University of Science and Technology, Danang, Vietnam in 2002 and 2008, respectively. She received the Ph.D. degree from the University of Electro-Communications, Tokyo, Japan under Japanese Government (Monbukagakusho) Scholarship in 2016. Dr. Quynh is currently a lecturer/researcher in The University of Danang - University of Science and Technology, Vietnam. Her research interests include high-speed optical communications and all-optical signal processing technologies.

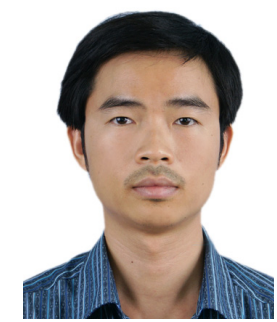

Ho Phuoc Tien received the Engineer degree in 2004 from Ho Chi Minh City University of Technology and the Master and Ph.D. degrees, in 2006 and 2010 respectively, from University of Grenoble. Dr. Tien is now a lecturer/researcher at The Univeristy of Danang - University of Science and Technology. His research interests include signal processing, image/video super resolution, visual attention and recognition.

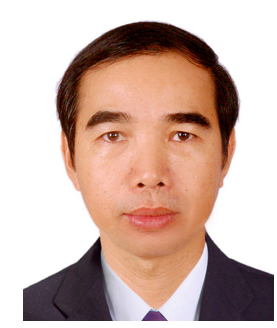

Nguyen Van Tuan was born in Danang, Vietnam, in 1963. He received the B.E. degree from The University of Danang-University of Science and Technology, Vietnam in 1985, and the M.E. and Ph.D. degrees from The Hanoi University of Science and Technology, Vietnam in 1998 and 2003, respectively. He worked as a postdoctoral fellow at the Department of Electronic and Communication, Sejong University, Korea. He is currently an Assosiate Professor at The University of Danang-University of Science and Technology where he works as the Dean of the Department of Electronic and Telecommunication Engineering.

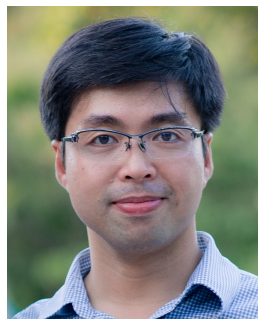

Nguyen Tan Hung was born in Danang, Vietnam, in 1980. He received the B.E. degree from The University of Danang-University of Science and Technology, Danang, Vietnam in 2003, and the M.E. and Ph.D. degrees from the University of Electro-Communications, Tokyo, Japan, in 2009 and 2012, respectively. From 2012 to 2016, he was a Researcher with the National Institute of Advanced Industrial Science and Technology, Tsukuba, Japan, where he worked on ultrafast and spectrally efficient all-optical network technologies, and development of an all-optical wavelength converter. He is currently a Teacher in the Department of Electronic and Telecommunication Engineering, The University of Danang-University of Science and Technology, Danang, Vietnam. His research interests include optical communications and networking, all-optical signal processing and photonic integrated circuits. 